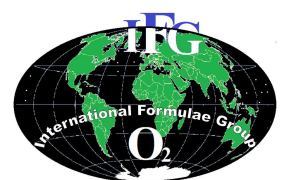

Available online at http://www.ifg-dg.org

Int. J. Biol. Chem. Sci. 10(3): 1153-1162, June 2016

International Journal

of Biological and

Chemical Sciences

ISSN 1997-342X (Online), ISSN 1991-8631 (Print)

Original Paper http://ajol.info/index.php/ijbcs http://indexmedicus.afro.who.int

\title{
Etude sur la pluviométrie d'une localité de la zone équatoriale de la RDC et relation avec la production du palmier à huile
}

\author{
Nuanisa LUYINDULA*, Nkebolo MANTANTU et Raymond BATANGA \\ Plantations et Huileries du Congo, Centre d'Etudes et d'Expérimentations en Agronomie Tropicale de \\ Yaligimba (Creaty), B.P. : 8611 Kinshasa I, RDC. \\ *Auteur correspondant ; E-mail : zeph.luyindula@feronia.com ; Tél. : +243810024 461
}

\begin{abstract}
RESUME
Le palmier à huile est l'une des cultures pérennes la plus répandue en Afrique Centrale. Une localité de la zone équatoriale de la RDC, où se trouve implantée une grande plantation agro-industrielle du palmier à huile, a ainsi été étudiée sur base de 40 années des données mensuelles et annuelles de précipitation. Cette pluviométrie a été analysée sur base de la quantité totale d'eau tombée, du nombre de jours de pluie et du déficit hydrique respectivement. Les paramètres considérés pour le palmier à huile ont été la production en régimes et l'extraction en huile de palme et se sont rapportés sur 8 années successives d'exploitation. De cette étude, il ressort une réduction significative du nombre des jours de pluie notée dans les 2 dernières décades (1990-1999, 2000-2009) par rapport aux 2 précédentes (1970-1979, 1980-1989), ce qui n'a pas été le cas pour la quantité totale d'eau tombée. Une corrélation négative hautement significative se dégage entre le taux d'extraction en huile de palme et la pluviométrie, en quantité d'eau tombée ou en nombre de jours de pluie. La production en régimes peut valablement être estimée sur base de la pluviométrie de 24 mois antérieurs. (C) 2016 International Formulae Group. All rights reserved.
\end{abstract}

Mots clés : Déficit hydrique, production en régimes, taux d'extraction, corrélation.

\section{Study of the rainfall of an area of equator region in DRC and relationship with oil palm production}

\begin{abstract}
The oil palm is one of the most planted perennial crops in Central Africa. One DRC locality of equator region where is implanted a big oil palm plantation has been analysed on base of 40 years of annual rainfall data. The total water, the number of rain days and the water deficit were the 3 rainfall parameters concerned. For the oil palm production, the fresh fruit bunch and oil extraction rate were also analysed. Compared to the first two decades (1970 -1979 and 1980-1999), the last two ones (1990-1999, 2000-2009) have shown significant reduction of number of rainfall days. A negative highly significant correlation was revealed between the oil extraction rate and the rainfall, expressed in water quantity or in number of rain days. The oil palm bunch production could be estimated on the base of 24 previous months of rainfall.

(C) 2016 International Formulae Group. All rights reserved.
\end{abstract}

Keywords: Water deficit, bunch production, extraction rate, correlation. 


\section{INTRODUCTION}

Les données climatiques constituent des informations importantes pour l'agronomie. En effet, l'eau, la température, l'ensoleillement sont autant des facteurs agissant sur la croissance des plantes et, plus particulièrement des cultures pérennes. Parmi les paramètres climatiques qui influent sur la production des cultures, la pluviométrie, plus précisément le bilan hydrique, occupe une place de première importance.

D'autre part, le palmier à huile, Elaeis guineensis Jacq., est l'une des cultures pérennes la plus répandue au monde, occupant plus de 7 millions d'hectares emblavés (Corley et Tinker, 2003). En Afrique de l'Ouest et Centrale, cet arbre est ainsi cultivé tant par les grandes sociétés agro-industrielles que par les petits planteurs et paysans. Toutefois, il joue un rôle non négligeable dans la déforestation et pourrait influencer les changements climatiques (Mezui et al., 2012; Aholoukpe et al., 2013). Cet effet sur l'environnement ne pourrait être apprécié qu'à travers l'évolution de la pluviométrie pour une région donnée.

En outre, plusieurs travaux réalisés sur le palmier à huile ont ainsi tenté de faire ressortir la relation assez étroite existant entre la pluviométrie et la production en régimes (Goh, 2000 ; Jacquemard, 2012; Ndoutoumou et al., 2014). Aussi, le déficit hydrique joue un rôle primordial sur la production en régimes chez le palmier à huile. Une période de sécheresse est suivie par des avortements et une réduction des inflorescences femelles et de sex-ratio sur le palmier, entraînant une baisse de la production en régimes 24 à 30 mois plus tard (Corley et Tinker, 2003).

Pour mieux apprécier l'influence de la précipitation sur quelques paramètres de production de palmier à huile, nous avons opté pour une approche systématique. Une telle approche se fonde sur les données climatiques fondamentales de plusieurs années mises en relation avec les performances d'une culture. Une relation éventuelle est ainsi mise en évidence grâce aux analyses statistiques de corrélation.
Dans le cadre de cette étude, les données sur la précipitation d'une localité de la zone équatoriale de la R.D.C. ont été analysées durant plusieurs décades pour mieux appréhender l'évolution de cet environnement particulier où se trouve implantée une grande société agroindustrielle. Nous avons tenté aussi de dégager les relations directes pouvant exister entre la pluviométrie et les paramètres de production du palmier à huile, plus précisément la production en régimes et le taux d'extraction en huile de palme.

\section{MATERIEL ET METHODES \\ Milieu d'étude}

Ce travail s'est réalisé à la localité de Yaligimba, située dans la zone équatoriale de la R.D.C., à $2^{\circ} 17 \mathrm{~N}, 22^{\circ} 51 \mathrm{E}, 435 \mathrm{~m}$ d'altitude et $1.400 \mathrm{~km}$ de l'océan atlantique, l'océan le plus proche. Dans cette localité se trouvent implantées les plantations de palmier à huile, disposant aussi d'une huilerie moderne. Le sol de cette région est un hydrokaolinitique ferralsol. $\mathrm{La}$ température moyenne journalière est de $25{ }^{\circ} \mathrm{C}$ tandis que l'insolation moyenne annuelle est de 1.810 heures d'ensoleillement (Luyindula et al., 2016) . La pluviométrie, par contre, fait partie de cette étude et a été analysée d'une manière très détaillée.

\section{Données météorologiques relatives à la pluviométrie}

Les précipitations mensuelles et annuelles d'une localité sont déterminées à l'aide des données des pluviomètres et exprimées en quantité d'eau tombée et en nombre de jours de pluie par mois ou année. Le bilan hydrique qui est une autre composante de la pluviométrie permet de préciser s'il y a eu réserve ou déficit hydrique dans le sol pour un végétal donné.

L'Institut de Recherches pour les Huiles et Oléagineux (IRHO) a proposé d'utiliser une formule simplifiée pour le calcul du bilan hydrique du palmier à huile (Corley et Tinker, 2003), qui est la suivante :

$$
B h=R+P m m-E T P
$$


où :

$B h=$ bilan hydrique du sol en mm d'eau (si $\mathrm{Bh}>0$, il y a une réserve hydrique dans le sol; si $\mathrm{Bh}<0$, il y a un déficit hydrique);

$R=$ réserve d'eau initiale du sol au début de la période considérée, cette période étant mensuelle dans cette étude. Le maximum mensuel pour R est fixé à $200 \mathrm{~mm}$;

Pmm = quantité d'eau de pluie récoltée (en $\mathrm{mm})$ pendant la période concernée ;

$E T P=$ évapotranspiration, fixée pour le palmier à huile sur base de nombre de jours de pluie (n); si $n>10: E T M=120 \mathrm{~mm}$, et si $\mathrm{n}<10: \mathrm{ETM}=150 \mathrm{~mm}$.

La pluviométrie a été également analysée par tranches de période de dix années ou décade. La décade 1952-1961 a été considérée comme décade de référence (DR) car elle a regroupé les 10 premières années de prélèvement de la pluviométrie à Yaligimba. Les données relatives aux années 1962-1969 sont inexistantes, ces années correspondant à la période de troubles qu'a connu cette zone. Aussi, nous n'avons étudié que la période de 1970 à 2009, soit 40 ans de données de précipitation, qui ont été comparées à la période de référence (DR).

\section{Paramètres de production de palmier à huile}

Les données de la production en régimes et ainsi que les taux d'extraction en huile de palme à l'usine proviennent de la plantation de PHC Yaligimba, une plantation avec environ 5.000 ha de palmier à huile en production durant la période de cette étude. L'étude s'est limitée aux années 1988 à 1995 (8 années de données de production d'huile de palme), le nombre d'hectares et l'âge moyen des palmiers en maturité étant relativement proches durant cette période. Cette restriction de la période considérée s'explique également par le fait des troubles qu'a connu cette région après l'année 1995, ayant entraîné l'arrêt de production pendant un peu plus d'une dizaine d'années.

Le palmier à huile est en production de régimes durant toute l'année de façon assez régulière, mais avec des pointes mensuelles suivant les régions. La variation mensuelle de la production en régimes au cours d'une année est ainsi exprimée en pourcentage de la production totale annuelle. Le taux d'extraction en huile de palme à l'usine est le rapport entre la quantité en poids d'huile de palme produite et le poids total des régimes usinés. Il s'exprime également en pourcentage.

\section{Analyse statistique des données \\ L'analyse des résultats de cette étude s'est basée sur certaines valeurs de statistique, principalement le coefficient de variation (CV) pour déterminer la variabilité des résultats de précipitations de plusieurs années et la corrélation simple $(r)$ pour apprécier la relation entre deux paramètres étudiés. Pour ce faire, les données ont été traitées sur Excel, puis analysées par le fichier statistique de Windows 10. Le test $t$ de Student a aussi été utilisé pour la comparaison des résultats de diverses décades, à l'aide du même fichier.}

\section{RESULTATS \\ Variations mensuelles et annuelles de la pluviométrie \\ Le Tableau 1 reprend les données} sur la pluviométrie annuelle moyenne de 40 années, regroupées en décades : décade 19701979 (D1), 1980-1989 (D2), 1990-1999 (D3) et 2000-2009 (D4). Ces résultats par décade sont comparés avec la décade de référence 1952-1961 (DR).

Aussi, la moyenne annuelle de la quantité totalité d'eau tombée durant les 40 années étudiées est de 1791 $225 \mathrm{~mm}$ (CV = $13 \%)$. Cette moyenne générale ainsi que les moyennes de pluviométrie par décade sont presque similaires à celle de DR (1761 mm).

En ce qui concerne le nombre total de jours de pluie, la moyenne annuelle des 40 années s'élève à $125 \pm 21 \mathrm{~mm}(\mathrm{CV}=17 \%)$. Par rapport à la décade de référence $(\mathrm{DR}=158$ $\mathrm{mm}$ ), on constate une diminution sensible du nombre de jours de pluie dans les autres décades. Cette réduction se situe à $88 \%, 89 \%$, $63 \%$ et $73 \%$, respectivement pour D1, D2, D3 et $\mathrm{D} 4$. En effet, les deux dernières décades se 
caractérisent par les plus faibles nombres de jours de pluie. Les CV du nombre de jours de pluie sont proches de ceux obtenus pour la quantité d'eau (Tableau 1). Les moyennes de répartition mensuelle des précipitations par décade sont données en quantité d'eau sur la Figure 1, et en nombre de jours de pluie sur la Figure 2. Toutefois, ces deux figures reprennent uniquement le DR comparé avec le D1 regroupé avec D2, et le D3 avec D4. Les mois successifs de décembre, janvier et février constituent les mois des plus faibles précipitations, avec mensuellement moins de $100 \mathrm{~mm}$ d'eau et marque donc une petite saison sèche, mais peu marquée, qui n'est nullement préjudiciable à la culture du palmier à huile. Le nombre de jours de pluie marque une nette baisse pour le D3-D4 par rapport au décade DR et D1-D2 pour tous les mois de l'année, comme on peut la noter clairement sur la Figure 2.

\section{Déficit hydrique}

Dans le microclimat de Yaligimba, la moyenne annuelle de déficit hydrique (Dh) de 30 ans (1980-2009) se situe à $158 \pm 100 \mathrm{~mm}$. Cependant, ce déficit montre une très grande variabilité $(\mathrm{CV}=63 \%)$. En effet, nul en 1999 et $2009(\mathrm{Dh}=0)$ et insignifiant en $1982(\mathrm{Dh}=$ 2), le Dh connait son maximum en 1997 et 2007 avec des valeurs respectivement de 328 et $331 \mathrm{~mm}$. Il est accepté que les meilleures zones de culture du palmier à huile sont celles où le déficit hydrique moyen annuel est inférieur ou égal à $200 \mathrm{~mm}$ (Goh, 2000 ; Corley et Tinker, 2003), ce qui est le cas de Yaligimba avec $75 \%$ des années étudiées (10 années sur 40 avec $\mathrm{Dh}>200 \mathrm{~mm}$ ).

\section{Relation entre les divers paramètres de la pluviométrie}

Le Tableau 2 reprend les coefficients de corrélation entre les divers paramètres de la pluviométrie: précipitations moyennes annuelles en $\mathrm{mm}$ (Pmm), nombre moyen de jours de pluie (NJP), déficit hydrique moyen annuel (Dh) et succession croissante des années ou mois (SCA/M). Les données annuelles de Pmm et NJP ne montrent pas de corrélation significative entre eux $\quad(r=0,01$ ns; ndl: 38). Par contre, une corrélation négative très hautement significative $(\mathrm{r}=$ $0,73^{* * *}$; ndl : 38 ) se dégage entre la SCA et le NJP, mais cette corrélation est non significative en ce qui concerne la relation entre la SCA et la Pmm. Les déficits hydriques (Dh) de 30 dernières années sont corrélés significativement avec les deux autres paramètres de pluviométrie, le Pmm ou le NJP. Par contre, aucune relation n'existe entre les Dh et les SCA, ce qui paraît évident.

\section{Production en régimes, taux d'extraction en huile et relation avec la pluviométrie}

Deux paramètres de production en huile de palme du palmier à huile ont été analysés : la production en régimes (PR) et le taux mensuel d'extraction en huile de palme $(\% \mathrm{TEH})$, obtenu après usinage des régimes.

\section{Production en régimes}

La production moyenne en régimes et sa répartition mensuelle sont reprises au Tableau 3 pour les 8 années concernées dans cette étude (1988-1995). Les mois de janvier et février ont donné les plus faibles productions, ne représentant que $4,8 \%$ et $5,8 \%$ de la production totale annuelle. Deux pics en haut de production s'observent, respectivement en avril et en octobre, avec $12,9 \%$ et $9,6 \%$ de la production annuelle, correspondant à un peu plus du double des mois de janvier ou février. Ces mêmes 2 pics sont également notés à la même période pour les précipitations mensuelles. Aussi, la production mensuelle en régimes et la précipitation mensuelle de 8 années consécutives (1988-1995) montrent une corrélation positive hautement significative ( $\mathrm{r}$ $=0,28^{* *}$; ndl : 94). Cette corrélation est négative, mais significative entre la production en régimes et le déficit hydrique (Dh) (Tableau 2).

Effet de la pluviométrie sur le taux d'extraction en huile de palme

Le Tableau 3 reprend également l'évolution mensuelle des taux d'extraction 
moyen en huile de palme (TEH\%) au cours de l'année (moyenne de 1988 à 1995). Les taux moyens varient du $17,17 \%$ pour la plus faible valeur mensuelle à $18,97 \%$ pour le taux le plus élevé, soit $10 \%$ supérieur par rapport au taux le plus bas. Il en ressort clairement que les mois de décembre, janvier et février, qui sont les mois de faible pluviométrie, se caractérisent par des taux d'extraction en huile de palme relativement plus élevés par rapport aux autres mois (mars à novembre). Les moyennes de taux d'extraction de ces 2 groupes sont de $18,81 \%$ pour les mois les plus secs et $18,12 \%$ pour les mois humides. L'analyse statistique par test $\mathrm{t}$ de Student, sur base des résultats annuels de 8 années concernées, dégage une différence hautement significative entre ces moyennes annuelles de ces 2 groupes $\left(t=3,75^{* *} ; \mathrm{dl}=6\right)$. D'autre part, une corrélation négative hautement significative ressort entre la Pmm et le TEH ( $\mathrm{r}$ $=-0,26^{* *}$; ndl : 94), et entre le NJP et le TEH ( $\mathrm{r}=-0,21 * ;$ ndl : 94). Aucune relation ne se dégage entre le TEH et la Dh.

\section{Relation entre la production en régimes et les précipitations antérieures}

Une certaine prédiction grossière de la production peut être réalisée sur base de la corrélation simple entre les productions réalisées et les précipitations antérieures mensuelles. Quatre périodes antérieures de précipitation ont été prises en considération par rapport à la production : 6, 12, 24, 30 mois avant la récolte. La meilleure corrélation ressort avec les précipitations en $\mathrm{mm}$ d'eau de 24 mois antérieurs à la récolte $(r=0,26 * *$; ndl: 96). Ce coefficient de corrélation est faible et non significative pour la période de 30 mois $(r=0,18)$. Par contre, cette corrélation est inexistante en considérant une période de 6 ou 12 mois avant la récolte $(\mathrm{r}=$ 0,07 ns et - 0,01 ns). Curieusement, contrairement aux précipitations exprimées en quantité d'eau tombée, les précipitations en nombre de jours de pluie se révèlent avec des meilleures coefficients de corrélation: $\mathrm{r}=$ $0,25^{*}$ pour 6 mois, $\mathrm{r}=0,45^{* * *}$ pour 12 mois, $\mathrm{r}=0,28^{* *}$ pour 24 mois et $\mathrm{r}=0,25^{*}$ pour 30 mois antérieurs à la récolte.

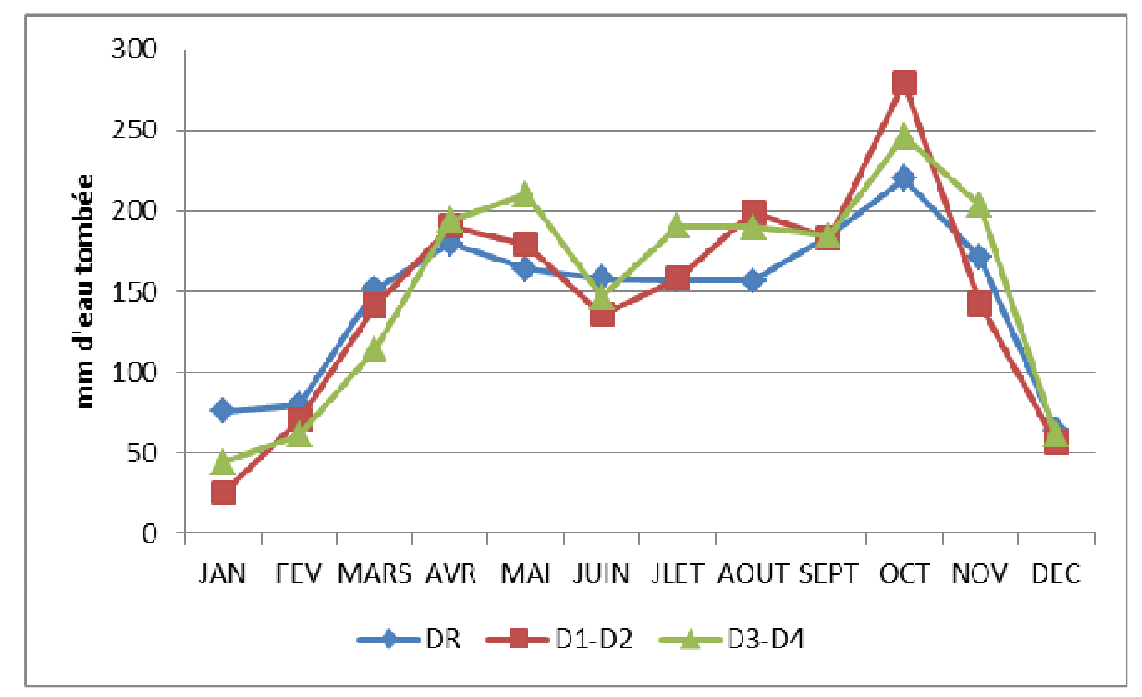

Figure 1: Pluviométries mensuelles en quantité d'eau tombée en mm pour DR (décade de référence). D1-D2 (bi-décade 1970-1989) et D3-D4 (bi-décade 1990-2009). 


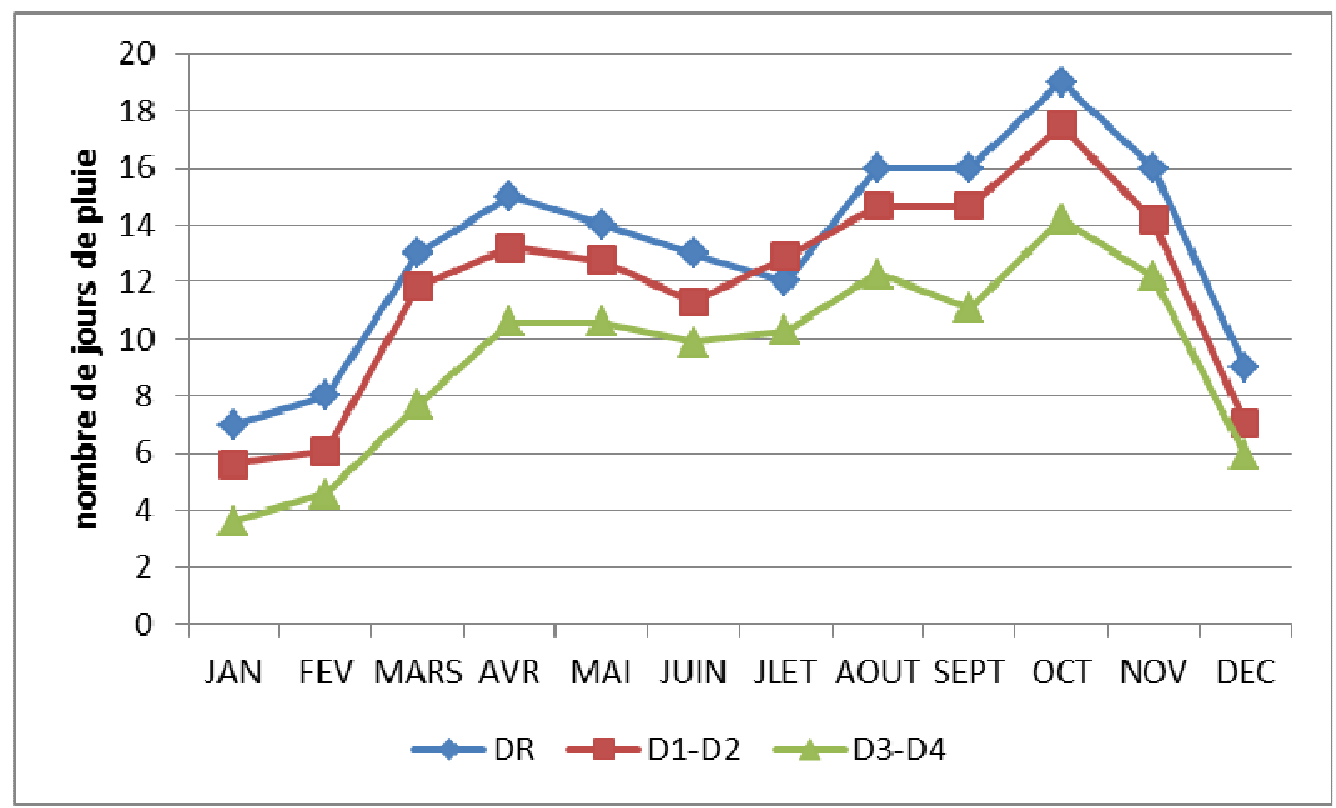

Figure 2 : Pluviométries mensuelles en nombre de jours de pluie de DR (décade de référence). D1D2 (bi-décade 1970-1989) et D3-D4 (bi-décade 1990-2009).

Tableau 1 : Précipitations annuelles en $\mathrm{mm}(\mathrm{P} \mathrm{mm})$, nombre de jours de pluies (NJP) et déficits hydriques (Dh) par décade à Yaligimba.

\begin{tabular}{lcccccc}
\hline Paramètres & DR & D1 & D2 & D3 & D4 & Moyenne \\
\hline P mm & 1.761 & 1.713 & 1.779 & 1.785 & 1.907 & 1.793 \\
Std & - & 197 & 216 & 266 & 227 & 227 \\
CV & - & 11 & 12 & 15 & 12 & 13 \\
\% DR & 100 & 97 & 101 & 101 & 108 & 102 \\
\hline NJP & 158 & 135 & 141 & 100 & 116 & 125 \\
Std & - & 11 & 11 & 14 & 14 & 21 \\
CV & - & 8 & 8 & 14 & 12 & 17 \\
\% DR & 100 & 88 & 89 & 63 & 73 & 79 \\
\hline Dh & - & - & 138 & 199 & 137 & 158 \\
Std & - & - & 90 & 102 & 104 & 100 \\
CV & - & - & 65 & 51 & 76 & 63 \\
\hline DR : décade de référence $1952-1961 ;$ D1 : décade 1970-1979; D2: décade 1980-1989; D3: décade 1990-1999; D4: \\
décade 2000-2009.
\end{tabular}


Tableau 2 : Coefficient de corrélation (r) entre divers paramètres.

\begin{tabular}{lcc}
\hline $\begin{array}{l}\text { Paramètres de } \\
\text { pluviométrie }\end{array}$ & $\begin{array}{c}\text { Données } \\
\text { annuelles de } \mathbf{4 0} \text { ans }\end{array}$ & $\begin{array}{c}\text { Données } \\
\text { mensuelles de } 8 \text { ans }\end{array}$ \\
\hline Pmm-NJP & $0,07 \mathrm{~ns}$ & $0,74^{* * *}$ \\
Pmm-Dh & $-0,65^{* * *}$ & $-0,55^{* * *}$ \\
Pmm-SCA/M & $0,34 * *$ & $0,00 \mathrm{~ns}$ \\
NJP-Dh & $-0,41^{* * *}$ & $-0,56 * * *$ \\
NJP-SCA/M & $-0,52^{* * *}$ & $-0,22^{* *}$ \\
Dh-SCA/M & $-0,08 \mathrm{~ns}$ & $-0,10 \mathrm{~ns}$ \\
Ndl & 38 & 96 \\
\hline Paramètres relatifs au & & \\
palmier à huile & Production régimes & Taux extraction huile \\
\hline Pmm & $0,28^{* *}$ & $-0,26 * *$ \\
NJP & $0,32 * *$ & $-0,21 *$ \\
Dh & $-0,24 *$ & $0,07 \mathrm{~ns}$ \\
PR & - & $-0,06 \mathrm{~ns}$ \\
Ndl & 96 & 96 \\
\hline Pmm: pluviométrie en mm d'eau; NPJ: pluviométrie en nombre jours de pluie; Dh: déficit hydrique; SCA/M: \\
succession croissante des 40 années (de 1970 à 2009) ou des mois durant 8 années (de 1988 à 1995); ndl: nombre de \\
dégrée de liberté.
\end{tabular}

Tableau 3 : Production en régimes (PR), variation de la production (VPR) et taux d'extraction en huile de palme (TEH) en relation avec la pluviométrie de la période 1988-1995.

\begin{tabular}{lccccc}
\hline Paramètres & PR (tonnes) & VPR \% & TEH \% & Pmm & NJP \\
\hline Janvier & 1.925 & 4,8 & 18,9 & 34,7 & 4 \\
Février & 2.326 & 5,8 & 18,5 & 65,5 & 5 \\
Mars & 3.848 & 9,6 & 18,5 & 133,6 & 10 \\
Avril & 5.175 & 12,9 & 18,3 & 187,3 & 12 \\
Mai & 4.099 & 10,2 & 18,3 & 199,9 & 12 \\
Juin & 3.559 & 8,9 & 18,4 & 135,1 & 10 \\
Juillet & 3.404 & 8,5 & 17,7 & 200,1 & 13 \\
Août & 2.490 & 6,2 & 18,1 & 179,1 & 12 \\
Septembre & 2.563 & 6,4 & 17,7 & 183,9 & 13 \\
Octobre & 3.833 & 9,6 & 18,2 & 240,4 & 15 \\
Novembre & 3.391 & 8,5 & 17,9 & 161,6 & 11 \\
Décembre & 3.484 & 8,7 & 18,9 & 61,6 & 5 \\
Total/Moy. & 40.097 & 100 & 18,3 & $1.782,8$ & 122 \\
\hline
\end{tabular}




\section{DISCUSSION}

L'étude de 40 années (1970 à 2009) de pluviométrie de la localité de Yaligimba fait ressortir une variation annuelle moyennement élevée pour la quantité totale d'eau tombée et le nombre de jours de pluie, le coefficient de variation étant de $12 \%$ et de $19 \%$ respectivement. Par contre, la variabilité est de loin plus grande pour le déficit hydrique $(\mathrm{CV}=63 \%)$. Pourtant, selon Nouffé et al. (2011), une tendance à la baisse de la pluviométrie est observée en Afrique Occidentale et Centrale dès la fin des années 60 jusqu'aux années 90 , ce qui n'a pas été confirmé dans la cuvette centrale congolaise, plus précisément dans le cas particulier du site de Yaligimba. Contrairement à toute attente, aucune corrélation significative n'a pu se dégager entre la pluviométrie annuelle et le nombre des jours de pluie correspondants. Les résultats similaires ont été signalés en Côte d'Ivoire et au Cameroun (IRHO, 1992). Par ailleurs, une diminution très significative de nombre de jours de pluie en fonction des années croissantes a été observée, alors qu'aucune relation n'a été dégagée pour la quantité d'eau tombée. Aussi, la pluviométrie ne devrait nullement être appréciée uniquement en fonction de la quantité d'eau tombée. Le nombre de jours de pluie, qui constitue un indice de la répartition d'eau des pluies, devrait également être considéré comme une donnée d'une grande valeur agronomique. Pourtant, très peu d'études mettent en exergue cette dernière donnée. Une diminution de nombres de pluies notée dans cet environnement, principalement pour les deux dernières décades, pourrait s'expliquer par le déboisement intense des environs de Yaligimba, à une cinquantaine de $\mathrm{km}$, depuis près d'une vingtaine d'années par une société forestière voisine. Cela a probablement occasionné des changements climatiques non négligeables, à l'origine de cette réduction sensible du nombre de jours de pluie. Par contre, la précipitation moyenne annuelle en quantité d'eau tombée demeure assez stable pendant cette même période. En Colombie, une diminution de la pluviométrie en $\mathrm{mm}$ d'eau de près de $20 \%$ a été noté suite à l'abattage de 3.700 ha de forêt pour la culture du palmier à huile. Toutefois, cette pluviométrie est ensuite revenue à son niveau initial, du fait de l'occupation du terrain par des jeunes palmiers et des plantes de couverture du sol (IRHO, 1992).

Une certaine similitude a été mise en évidence entre les précipitations moyennes mensuelles et la production en régimes du palmier à huile, les mois de faibles pluviométries correspondant aussi aux mois de faibles productions (décembre, janvier et février). Toutefois, selon Goh (2000) et Corley et Tinker (2003), les variations de la production d'une année devraient être attribuées aux fluctuations de la précipitation de 10-11 et 20-24 mois précédents. Cela n'a pu être confirmé dans le cadre de cette étude seulement pour la période antérieure de 24 mois. Il est vrai que cette période correspond à la différentiation sexuelle, qui conditionne principalement la production en régimes attendue. Des corrélations positives significatives se sont dégagées entre la production en régimes à tous les stades considérés, et spécialement pour la période de 12 mois.

La relation entre la production en régimes et la pluviométrie antérieure, bien connue en Asie du Sud-Est, n'a pu être totalement confirmée dans l'environnement de Yaligimba. Dans la zone équatoriale de la RDC où la saison sèche est très peu marquée, le nombre de jours de pluie serait un élément prépondérant de la pluviométrie. Ce qui n'est jamais relevé dans divers travaux consultés, où la pluviométrie est appréciée uniquement en quantité d'eau.

Par ailleurs, d'une manière générale, les taux d'extraction en huile de palme ont été 
assez bas suite à la vétusté de l'usine de Yaligimba durant cette époque. Des taux de plus de $22 \%$ sont normalement réalisés dans plusieurs huileries modernes (Rajanaidu et al., 2005; Jacquemard, 2012). Quant à la variation mensuelle de taux d'extraction en huile de palme en fonction de la pluviométrie, les mois de faible pluviométrie se caractérisent par des taux d'extraction en huile de palme relativement plus élevés par rapport aux autres mois. Ce phénomène serait largement dû à l'action directe de l'eau de pluie. En effet, les précipitations ont pour conséquence la hausse de l'humidité de régimes et, par conséquent, une certaine augmentation du poids des régimes, mais également une perte plus importante en fruits détachés en plantation non ramenés totalement à l'usine, ce qui affecterait alors le taux d'extraction en huile de palme.

\section{Conclusion}

Il est évident que les effets de la pluviométrie ne peuvent être totalement dissociés des autres éléments climatiques qui lui sont largement liés. Néanmoins, il nous a semblé raisonnable, dans le cadre de cette étude, de considérer la pluviométrie séparément des autres paramètres afin de faciliter l'analyse du problème et son interprétation. Les précipitations en quantité d'eau tombée à Yaligimba demeurent presque stables durant les 40 années analysées alors que les nombres de jours de pluie diminuent significativement. Une relation très nette a également été mise en évidence entre les précipitations moyennes mensuelles et le taux moyen mensuel d'extraction en huile de palme. En outre, seule la prédiction de la production basée sur 24 mois antérieure de pluviométrie en quantité d'eau a pu être mise en évidence. Cette prévision semble toutefois meilleure en considérant la pluviométrie en nombre de jours de pluie.

\section{CONTRIBUTIONS DES AUTEURS}

L'étude a été proposée et réalisée par $\mathrm{NL}$, tandis que NM et RB ont contribué par des suggestions très constructives sur l'interprétation des résultats.

\section{CONFLIT D'INTERÊTS}

Les auteurs déclarent qu'il n'y a aucun conflit d'intérêt dans le cadre de cet article.

\section{REMERCIEMENTS}

Les auteurs expriment leurs sincères remerciements au Chief Executive Officer du groupe Feronia, Mr Xavier de Carnière, pour ses nombreux encouragements dans la relance de la recherche agronomique au sein du groupe Feronia. Leurs remerciements s'adressent également aux travailleurs de Creaty qui ont participé dans la collecte des données.

\section{REFERENCES}

Aholoukpe H, Vissoh VP, Amadji G, Deleporte P, Dubois B Nodichao L, Glele Kakaï R, Chote JL, Blavet D. 2013. Typologie des plantations villageoises de palmier à huile dans le département de Plateau au Bénin. Int. J. Biol. Chem. Sci., 7(3): 978-999. DOI : http://dx.doi.org/10.4314/ijbcs.v7i3.8.

Corley RHV, Tinker PB. 2003. The Oil Palm ( $4^{\text {nd }}$ edn ). Blackwell Publishing: Oxford.

Goh KJ. 2000. Climatic requirements of the oil palm for high yields. In Managing Oil Palm for High Yields: Agronomic Principles, Goh KJ (ed). Malaysian Soc. of Soil Sc. and Param Agric. Surveys: Kuala Lumpur; 1-17.

IRHO. 1992. Rapport d'activité 1989-1991 de l'Institut de Recherches pour les Huiles et Oléagineux (IRHO - CIRAD). Oléagineux, 47(6) : 266-456.

Jacquemard JC. 2012. Le Palmier à Huile. Agricultures Tropicales en Poche. CTA, 
Presses Agronomiques de Gembloux: Gembloux.

Luyindula N, Mantantu N, Batanga R, Bois d'Enghien P. 2016. Some observations on albo-nigrescens, albo-virescens and virescens types of oil palm planted at Yaligimba (DRC). WJAR, 4(4): 114118. DOI : 10.12691/wjar-4-4-3.

Maraux F. 2002. Le climat et la production végétale. In Mémento de l'Agronomie, Ministre des Affaires Etrangères, CIRAD, GRET : Paris ; 443-446.

Mezui AMR, Mweru MJP, Tchouamo IR. 2012. Impact du développement des palmeraies familiales sur la déforestation et dans les ménages du Cameroun. Int. J. Biol. Chem. Sci., 6(6): 30173026. DOI: http://dx.doi.org/10.4314/ijbcs.v6i6.4.
Ndoutoumou PN, Ondo-Azi AS, Ovono PO, Nkoume Mba D, Nkodo F. 2014. Essai d'optimisation de la production du palmier à huile (Elaeis guineensis J.) sur base des données climatiques dans la région du Sud-Ouest Cameroun. Afrique Science, 10(1): 208- 216.

http://www.afriquescience.info.

Rajanaidu N, Kushairi A Din, Isa M, Maizura I, Noh A. 2005. Oil palm planting materials: current developments and competitiveness with other oil bearing crops. In PIPOC 2005 Int. Palm Oil Cong.-Agric., Biotech. \& Sustain. Conf. MPOB : Kuala Lumpur ; 645-659. 